\title{
Nonlinear Brownian Motion and Higgs Mechanism
}

\author{
Alexander Glück \& Helmuth Hüffel \\ Faculty of Physics, University of Vienna \\ 1090 Vienna, Austria
}

\begin{abstract}
An extension of the stochastic quantization scheme is proposed by adding nonlinear terms to the field equations. Our modification is motivated by the recently established theory of active Brownian motion. We discuss a way of promoting this theory to the case of infinite degrees of freedom. Equilibrium distributions can be calculated exactly and are interpreted as path integral densities of quantum field theories. By applying our procedure to scalar QED, the symmetry breaking potential of the Higgs mechanism arises as the equilibrium solution.
\end{abstract}

\section{Introduction}

At the beginning we want to give a short review of the interconnection between Brownian motion and Stochastic Quantization. The dynamical evolution of the position $\mathbf{q}$ of a Brownian particle is described by the Langevin equation $([1,[2])$

$$
\frac{d q^{i}(t)}{d t}=-\delta^{i j} \frac{\partial U(q)}{\partial q^{j}}+\eta^{i}(t)
$$

with

$$
\left\langle\eta^{i}(t)\right\rangle=0 \quad, \quad\left\langle\eta^{i}(t) \eta^{j}(\bar{t})\right\rangle=2 \delta^{i j} \delta(t-\bar{t})
$$

and $U(q)$ being the external potential. The "noise"-variable $\eta$ represents the random impacts on the particle, resulting from the molecules of the liquid, in which the particle is suspended. This corresponds to the correlation relation (2), from which follows that $\eta$ has infinite variance. The evolution of the probability distribution $\rho(q, t)$ is given by the Smoluchowski equation

$$
\frac{\partial \rho(q, t)}{\partial t}=\frac{\partial}{\partial q^{i}} \delta^{i j}\left(\frac{\partial U(q)}{\partial q^{j}}+\frac{\partial}{\partial q^{j}}\right) \rho(q, t) .
$$

Solving this equation for $\frac{\partial \rho}{\partial t}=0$ defines an equilibrium distribution, which is

$$
\rho_{\text {equ }}(q) \sim e^{-U(q)},
$$

so that equilibrium averages for a function $f(q)$ are calculated as

$$
\langle f(q)\rangle_{e q u} \sim \int d^{n} q e^{-U(q)} f(q) .
$$


Stochastic dynamics leading to the equilibrium solution (4) can be formulated also in phase space, by imposing the Langevin equations

$$
\frac{d q^{i}(t)}{d t}=\frac{\partial H(q, p)}{\partial p_{i}(t)} \quad, \quad \frac{d p_{i}(t)}{d t}=-\frac{\partial H(q, p)}{\partial q^{i}(t)}-\delta_{i j} \frac{\partial H(q, p)}{\partial p_{j}(t)}+\eta_{i}
$$

with

$$
\left\langle\eta_{i}(t)\right\rangle=0 \quad, \quad\left\langle\eta_{i}(t) \eta_{j}(\bar{t})\right\rangle=2 \delta_{i j} \delta(t-\bar{t})
$$

and $H(q, p)=\frac{p^{2}}{2}+U(q)$. The corresponding Fokker Planck equation for the distribution $\rho(q, p)$ has the equilibrium solution

$$
\rho_{\text {equ }}(q, p) \sim e^{-\frac{p^{2}}{2}-U(q)}=e^{-H(q, p)} .
$$

Hence, the equilibrium average for a function $f(q, p)$ is given by

$$
\langle f(q, p)\rangle_{e q u} \sim \int d^{n} q d^{n} p e^{-H(q, p)} f(q, p) .
$$

For functions $f=f(q)$, the momentum integral can be carried out by Gaussian integration, so that

$$
\langle f(q)\rangle_{e q u} \sim \int d^{n} q e^{-U(q)} f(q)
$$

which is identical to (5). The idea of Stochastic Quantization $([3],[4,[5])$ is to view the path integral density $e^{-S[\phi]}$ as the equilibrium distribution of a specific stochastic process, just as $e^{-U(q)}$ is the equilibrium distribution of the stochastic process (1). The field theoretic generalization of equation (1) is

$$
\frac{\partial \phi(x, s)}{\partial s}=-\frac{\delta S}{\delta \phi}(x, s)+\eta(x, s)
$$

where $x=(t, \mathbf{x}) . S=S[\phi]$ denotes the Euclidean action of the theory. The noise correlations are

$$
\langle\eta(x, s)\rangle=0 \quad, \quad\langle\eta(x, s) \eta(\bar{x}, \bar{s})\rangle=2 \delta^{4}(x-\bar{x}) \delta(s-\bar{s}) .
$$

Fields evolve in the parameter $s$ ('stochastic time'), which is the evolution parameter of the stochastic process. Equation (11) defines a field equation for a five dimensional classical field theory, which differs from the usual field equations $\frac{\delta S}{\delta \phi}=0$ by the existence of the noise field $\eta(x, s)$ and the evolution in the stochastic time.

The Smoluchowski equation for the probability distribution $\rho[\phi, s]$ corresponding to the Langevin equation (11) is

$$
\frac{\partial \rho[\phi, s]}{\partial s}=\int d^{4} x \frac{\delta}{\delta \phi}\left(\frac{\delta S[\phi]}{\delta \phi}+\frac{\delta}{\delta \phi},\right) \rho[\phi, s]
$$


whose equilibrium solution can be read of directly:

$$
\rho_{\text {equ }}[\phi] \sim e^{-S[\phi]} .
$$

Quantum field theory Green functions can thus be interpreted as the equilibrium limit of stochastic averages:

$$
G\left(x_{1}, \ldots, x_{n}\right)=\int \mathcal{D} \phi \rho_{\text {equ }}[\phi] \phi\left(x_{1}\right) \ldots \phi\left(x_{n}\right) .
$$

\section{Active Brownian Motion in Phase Space}

A possible extension of the Brownian motion model is given by introducing nonlinear terms in the dynamical equations. Nonlinear Brownian Motion is particulary described by the theory of so-called active Brownian motion, which was originally formulated in phase space $([6],[7],[8],[9])$. Active Brownian particles differ from ordinary Brownian particles by an additional internal degree of freedom $e$, which is interpreted as a form of internal energy. The $2 n$-dimensional phase space is extended by introducing a new internal degree of freedom $e$, which satisfies the following dynamical equation:

$$
\frac{d e}{d t}=c_{1}-c_{2} e-c_{3} e \frac{p^{2}}{2}
$$

The parameters $c_{1}, c_{2}, c_{3}$ are assumed to be constant and positive. A physical motivation for the equation above can be given in terms of biological systems. $c_{1}$ represents a constant intake of energy, the second term describes a loss of energy due to internal processes, and the last term models the coupling of $e$ to the momentum, in the form of a conversion of internal energy into kinetic energy. Active Brownian particles can thus be viewed as organisms with an internal energy depot, which is increased by taking up food (first term), and decreased by internal processes of the organism (second term) and conversion of internal energy into motion (third term).

Stochastic dynamics in the extended phase space are closed by specifying a coupling of the coordinates $(\mathbf{q}, \mathbf{p})$ to the internal energy:

$$
\begin{gathered}
\frac{d q^{i}}{d t}=\frac{\partial H}{\partial p_{i}} \\
\frac{d p_{i}}{d t}=-\frac{\partial H}{\partial q^{i}}-\delta_{i j} \frac{\partial H}{\partial p_{j}}+c e \delta_{i j} \frac{\partial H}{\partial p_{j}}+\eta_{i}
\end{gathered}
$$

with

$$
\left\langle\eta_{i}(t)\right\rangle=0 \quad, \quad\left\langle\eta_{i}(t) \eta_{j}(\bar{t})\right\rangle=2 \delta_{i j} \delta(t-\bar{t})
$$

where $H=\frac{p^{2}}{2}+U(q)$ and $c>0$. Assuming that the evolution of the internal energy takes place on an essentially shorter time scale than that of the other 
coordinates, we can set $\frac{d e}{d t}=0$ and thus get an explicit depence of the internal energy on the momentum:

$$
e=e(p)=\frac{c_{1}}{c_{2}+c_{3} \frac{p^{2}}{2}} .
$$

Hence, the internal energy can be eliminated from the dynamical equations and we are left with stochastic dynamics that represent a system with a momentum dependent friction function:

$$
\begin{gathered}
\frac{d q^{i}}{d t}=\frac{\partial H}{\partial p_{i}} \\
\frac{d p_{i}}{d t}=-\delta_{i j} \frac{\partial H}{\partial q^{i}}-\gamma(p) \delta_{i j} \frac{\partial H}{\partial p_{j}}+\eta_{i} \\
\gamma(p)=1-c \frac{c_{1}}{c_{2}+c_{3} \frac{p^{2}}{2}} .
\end{gathered}
$$

Given the assumption that the coupling of $e$ to the kinetic energy is small $\left(c_{3} \ll 1\right)$, an expansion to first order in $p^{2}$ yields $\gamma(p) \simeq \gamma_{1}+\gamma_{2} p^{2}$ with $\gamma_{1} \equiv \gamma_{0}-c \frac{c_{1}}{c_{2}}$ and $\gamma_{2} \equiv c \frac{c_{1} c_{3}}{2 c_{2}^{2}}$. $\quad \gamma_{1}$ and $\gamma_{2}$ can be chosen independently of each other. $\gamma_{2}$ is strictly positive, whereas $\gamma_{1}$ can be chosen to be negative. Explicit computations of equilibrium solutions in phase space are done by specifying the potential $U(q)$ and by carrying out a certain avering procedure, which shows up to be necessary due to the nonlinear nature of the equations. Given the case of a harmonic potential and one degree of freedom: $H=\frac{p^{2}}{2}+w^{2} \frac{q^{2}}{2}$, the stochastic differential equations are

$$
\frac{d q}{d t}=p \quad, \quad \frac{d p}{d t}=-w^{2} q-\left(\gamma_{1}+\gamma_{2} p^{2}\right) p+\eta .
$$

Transforming via the Ito formula to action-angle variables $(H, \phi)$ :

$$
q(H, \phi)=\frac{\sqrt{2 H}}{w} \cos \phi \quad, \quad p(H, \phi)=\sqrt{2 H} \sin \phi
$$

and taking averages with respect to $\phi$, an averaged Fokker Planck equation for the distribution $\rho[H, t]$ can be obtained:

$$
\frac{d \rho}{d t}=\frac{\partial}{\partial H}\left(\gamma_{1} H+\frac{3}{2} \gamma_{2} H^{2}+H \frac{\partial}{\partial H}\right) \rho,
$$

whose equilibrium solution reads

$$
\rho_{\text {equ }}(H) \sim e^{a H-b H^{2}}
$$

with $a \equiv-\gamma_{1}>0$ and $b \equiv \frac{3}{4} \gamma_{2}>0$ being the independent parameters of the theory. It is remarkable that there is no direct way of generalizing this result 
to higher degrees of freedom. In [10], the result for $n=2$ was computed to be

$$
\rho_{\text {equ }}\left(H_{1}, H_{2}, \theta\right) \sim e^{a\left(H_{1}+H_{2}\right)-b\left\{\left(H_{1}\right)^{2}+\left(H_{2}\right)^{2}\right\}+\frac{4}{3} b H_{1} H_{2}\left\{1+\frac{1}{2} \cos (2 \theta)\right\}},
$$

where $H_{i}=\frac{p_{i}^{2}}{2}+w^{2} \frac{q^{2}}{2} ; a, b>0$ and $\theta$ being an angle variable coming from the avering procedure.

\section{Active Brownian Motion in Configuration Space}

We now give a new formulation of active Brownian motion in $\mathbf{q}$-space and calculate an exact equilibrium distribution. Whereas in the phase space formalism a coupling of the internal energy to the momentum was proposed, we now specify a coupling to the position q. A natural choice is to impose a coupling of $e$ to the potential energy $U(q)$, in analogy to the formalism in phase space, where a coupling of the internal energy to the kinetic energy was stated. We will work with a general potential $U(q)$ in what follows, which is a main difference to the calculations in phase space, where the potential has to be specified in order to calculate an approximative equilibirum distribution. In analogy to the situation in phase space, where $e$ was coupled to the momentum-gradient of the mechanical energy, we now postulate a coupling of $e$ to the $\mathbf{q}$-gradient of $H(\mathbf{q}, \mathbf{p})$. Stochastic dymanics are given by the following equations:

$$
\begin{gathered}
\frac{d q^{i}}{d t}=-\delta^{i j} \frac{\partial U}{\partial q^{j}}+c e \delta^{i j} \frac{\partial U}{\partial q^{j}}+\eta^{i} \\
\frac{d e}{d t}=c_{1}-c_{2} e-c_{3} e U(q)
\end{gathered}
$$

with

$$
\left\langle\eta^{i}(t)\right\rangle=0 \quad, \quad\left\langle\eta^{i}(t) \eta^{j}(\bar{t})\right\rangle=2 \delta^{i j} \delta(t-\bar{t}) .
$$

$c, c_{1}, c_{2}, c_{3}$ are assumed to be positive constants. Elimination of $e$ allows us to calculate an exact equilibrium solution of the Fokker Planck equation. We set $\frac{d e}{d t}=0$, so that $e$ is expressed in terms of the absolute value of the position:

$$
e=e(q)=\frac{c_{1}}{c_{2}+c_{3} U(q)} .
$$

Plugging this into (29) shows that the coupling term can be written as a gradient. Therefore, the Langevin equation is in the standard form

$$
\frac{d q^{i}}{d t}=-\delta^{i j} \frac{\partial \tilde{U}}{\partial q^{j}}+\eta^{i}
$$

with the effective potential

$$
\tilde{U}(q)=U(q)-c \frac{c_{1}}{c_{3}} \ln \left\{c_{2}+c_{3} U(q)\right\}
$$


The Smoluchowski equation reads

$$
\frac{d \rho}{d t}=\frac{\partial}{\partial q^{i}} \delta^{i j}\left(\frac{\partial \tilde{U}}{\partial q^{j}}+\frac{\partial}{\partial q^{j}}\right) \rho,
$$

which has the equilibrium solution

$$
\rho_{\text {equ }}(q) \sim e^{-\tilde{U}(q)}=e^{-U(q)+c \frac{c_{1}}{c_{3}} \ln \left\{c_{2}+c_{3} U(q)\right\}} .
$$

There are three remarks:

- No averaging procedure was needed to find an equilibrium distribution, like in the previous phase space formalism.

- The result is valid for a general dimension $n$. We have mentioned the fact that in the phase space formalism, generalizations of the one dimensional case to higher dimensions are nontrivial. In the present formulation, we do not have to restrict ourselves to lower dimensional cases.

- The above computations hold for a general potential $U(q)$, whereas in the action-angle based averaging procedure in phase space the potential has to be specified explicitly.

Factorizing the logarithm in (36) and absorbing the factor $\ln \left(c_{2}\right)$ into the normalization, we expand the remaining term under the assumption that the coupling of $e$ to the potential energy is small compared to internal dissipation $\left(\frac{c_{3}}{c_{2}}\right.$ small). By choosing $1-c \frac{c_{1}}{c_{3}}<0$ and with the definitions $a \equiv-\left(1-c \frac{c_{1}}{c_{3}}\right)$ and $b \equiv \frac{1}{2} c \frac{c_{1} c_{3}}{\left(c_{2}\right)^{2}}$, the effective potential reads

$$
\tilde{U}(q)=a U(q)-b U(q)^{2}
$$

with $a, b>0$ being the independet parameters of the theory. Given the case of one dimension and by assuming a harmonic potential $U(q)=w^{2} \frac{q^{2}}{2}, \tilde{U}(q)$ has two minima at $q_{0 \pm}= \pm \frac{1}{w}\left(\frac{a}{b}\right)^{\frac{1}{2}}$. Studying this theory near one of the minima yields the classical mechanism of symmetry breaking, which means that active Brownian motion in configuration space under the assumption of a harmonic external potential gives rise to an equilibrium distribution with broken symmetry.

\section{Nonlinear Stochastic Quantization}

We set up a field theory generalization of active Brownian motion in configuration space and calculate an exact equilibrium distribution. When applied to scalar QED, this modified stochastic quantization procedure leads to the 
symmetry breaking potential of the Higgs mechanism.

Equations (29)-(31) for active Brownian motion in configuration space are generalized to

$$
\begin{gathered}
\frac{\partial \phi^{i}(x, s)}{\partial s}=-\delta^{i j} \frac{\delta S}{\delta \phi^{j}}(x, s)+c e \delta^{i j} \frac{\partial V}{\partial \phi^{j}}(x, s)+\eta^{i}(x, s) \\
\frac{\partial e}{\partial s}=c_{1}-c_{2} e-c_{3} e V(\phi) \\
\left\langle\eta(x, s)^{i}\right\rangle=0 \quad, \quad\left\langle\eta(x, s)^{i} \eta(\bar{x}, \bar{s})^{j}\right\rangle=2 \delta^{i j} \delta^{4}(x-\bar{x}) \delta(s-\bar{s})
\end{gathered}
$$

for a collection of fields $\left\{\phi^{i}\right\}=\phi$, where $S=S[\phi]$ denotes the Euclidean action and the constants $c, c_{1}, c_{2}, c_{3}$ are assumed to be positive. Setting $\frac{\partial e}{\partial s}=0$, we get an expression of $e$ in terms of the fields:

$$
e=e(\phi)=\frac{c_{1}}{c_{2}+c_{3} V(\phi)} .
$$

Plugging this into (38), the field equations can be written in the standard form

$$
\frac{\partial \phi^{i}(x, s)}{\partial s}=-\delta^{i j} \frac{\delta \tilde{S}}{\delta \phi^{j}}(x, s)+\eta^{i}(x, s)
$$

with the effective action

$$
\tilde{S}[\phi] \equiv \int d^{4} x\left\{\mathcal{L}(\phi, \partial \phi)-\frac{c c_{1}}{c_{3}} \ln \left(c_{2}+c_{3} V(\phi)\right)\right\}=\int d^{4} x \tilde{\mathcal{L}}(\phi, \partial \phi) .
$$

The effective Lagrangian $\tilde{\mathcal{L}}$ is analogous to the effective potential (34). The Smoluchowski equation corresponding to (42) reads

$$
\frac{\partial \rho[\phi, s]}{\partial s}=\int d^{4} x \frac{\delta}{\delta \phi^{i}} \delta^{i j}\left(\frac{\delta \tilde{S}}{\delta \phi^{j}}+\frac{\delta}{\delta \phi^{j}}\right) \rho[\phi, s],
$$

which has the equilibrium solution

$$
\rho_{\text {equ }}[\phi] \sim e^{-\tilde{S}[\phi]} .
$$

Carrying out the expansion of the effective Lagrangian in the same way as we did in the case of finite degrees of freedom leads to an effective field theory potential of the form

$$
\tilde{V}(\phi)=-a V(\phi)+b V(\phi)^{2}
$$

with $a=-\left(1-c \frac{c_{1}}{c_{2}}\right)>0$ and $b=\frac{1}{2} c \frac{c_{1} c_{3}}{\left(c_{2}\right)^{2}}>0$. Equilibrium Green functions are then given by

$$
G\left(x_{1}, \ldots, x_{n}\right) \sim \int \mathcal{D} \phi e^{-\int d^{4} x\left\{\frac{1}{2}(\partial \phi)^{2}-a V(\phi)+b V(\phi)^{2}\right\}} \phi\left(x_{1}\right) \ldots \phi\left(x_{n}\right)
$$


and are identified with the Green functions of a quantized field theory. Applying the general formalism outlined above in the case of a free, real scalar field demonstrates how symmetry breaking immediately drops out of the nonlinear stochastic quantization scheme. The Euclidean Lagrangian

$$
\mathcal{L}(\phi, \partial \phi)=\frac{1}{2}(\partial \phi)^{2}+\frac{m^{2}}{2} \phi^{2}
$$

leads to the equilibrium distribution

$$
\rho_{\text {equ }}[\phi] \sim e^{-\int d^{4} x\left\{\frac{1}{2}(\partial \phi)^{2}-a \frac{m^{2}}{2} \phi^{2}+b \frac{m^{4}}{4} \phi^{4}\right\}}
$$

with $a, b>0$. Identifying the effective potential

$$
\tilde{V}(\phi)=-\frac{a}{2} m^{2} \phi^{2}+\frac{b}{4} m^{4} \phi^{4}
$$

and its minima

$$
\phi_{0_{ \pm}}= \pm \frac{1}{m}\left(\frac{a}{b}\right)^{\frac{1}{2}}
$$

the theory can be studied near one of its minima in the usual way. Having this simple example in mind, we can proceed to the construction of the Higgs mechanism via the application of the nonlinear stochastic quantization scheme to scalar QED. The Euclidean Lagrangian

$$
\mathcal{L}=\frac{1}{4} F_{\mu \nu} F^{\mu \nu}+\left(D_{\mu} \phi\right)^{*}\left(D^{\mu} \phi\right)+V(|\phi|) \quad, \quad V(|\phi|)=m^{2} \phi^{*} \phi
$$

$(D=\partial-i A)$ leads to the following stochastic differential equations:

$$
\begin{aligned}
\frac{\partial A_{\mu}}{\partial s} & =\partial^{\nu} F_{\nu \mu}-i\left(\phi^{*} \partial_{\mu} \phi-\phi \partial_{\mu} \phi^{*}\right)-2 \phi^{*} \phi A_{\mu}+\xi_{\mu} \\
\frac{\partial \phi}{\partial s} & =\left\{\left(D_{\mu} D^{\mu}\right)^{*}-m^{2}\right\} \phi^{*}+c e m^{2} \phi^{*}+\eta \\
\frac{\partial \phi^{*}}{\partial s} & =\left\{D_{\mu} D^{\mu}-m^{2}\right\} \phi+c e m^{2} \phi+\eta^{*} \\
\frac{\partial e}{\partial s} & =c_{1}-c_{2} e-c_{3} e m^{2} \phi^{*} \phi
\end{aligned}
$$

with the noise correlations

$$
\begin{gathered}
\langle\eta(x, s)\rangle=0 \quad, \quad\left\langle\eta(x, s)^{*}\right\rangle=0 \quad, \quad\left\langle\xi_{\mu}(x, s)\right\rangle=0 \\
\left\langle\eta(x, s) \eta(\bar{x}, \bar{s})^{*}\right\rangle=2 \delta^{4}(x-\bar{x}) \delta(s-\bar{s}) \\
\left\langle\xi_{\mu}(x, s) \xi_{\nu}(\bar{x}, \bar{s})\right\rangle=2 \delta_{\mu \nu} \delta^{4}(x-\bar{x}) \delta(s-\bar{s})
\end{gathered}
$$

Elimination of the internal energy finally allows us to write this set of equations in the form

$$
\frac{\partial \varphi^{i}(x, s)}{\partial s}=-\delta^{i j} \frac{\delta \tilde{S}}{\delta \varphi^{j}}(x, s)+\chi^{i}(x, s)
$$


with

$$
\left\langle\chi^{i}(x, s)\right\rangle=0 \quad, \quad\left\langle\chi^{i}(x, s) \chi^{j}(\bar{x}, \bar{s})\right\rangle=2 \delta^{i j} \delta^{4}(x-\bar{x}) \delta(s-\bar{s})
$$

where $\varphi=\left(\phi, \phi^{*}, A\right)$ and $\chi=\left(\eta, \eta^{*}, \xi\right)$. Approximating the effective action like in the previous examples yields

$$
\tilde{S}[\varphi]=\int d^{4} x\left\{\frac{1}{4} F_{\mu \nu} F^{\mu \nu}+\left(D_{\mu} \phi\right)^{*}\left(D^{\mu} \phi\right)-a m^{2}|\phi|^{2}+b m^{4}|\phi|^{4}\right\} .
$$

In complete analogy to the stochastic quantization procedure for QED ([1]) a gauge fixed equilibrium distribution can be obtained straightforwardly:

$$
\rho_{\text {equ }} \sim e^{-\int d^{4} x \tilde{\mathcal{L}}_{g f}},
$$

where the gauge fixed effective Lagrangian reads

$$
\tilde{\mathcal{L}}_{g f}=\frac{1}{4} F_{\mu \nu} F^{\mu \nu}+\frac{1}{2}(\partial A)^{2}+\left(D_{\mu} \phi\right)^{*}\left(D^{\mu} \phi\right)-a m^{2}|\phi|^{2}+b m^{4}|\phi|^{4} .
$$

The effective potential

$$
\tilde{V}(|\phi|)=-a m^{2}|\phi|^{2}+b m^{4}|\phi|^{4}
$$

has two minima at

$$
|\phi|_{0_{ \pm}}= \pm \frac{1}{m}\left(\frac{a}{2 b}\right)^{\frac{1}{2}}
$$

Shifting the Lagrangian, the mass of the gauge field and the Higgs mass are identified:

$$
m_{A}^{2}=\frac{a}{b}\left(\frac{1}{m}\right)^{2} \quad, \quad m_{H}^{2}=2 a m^{2}
$$

\section{Conclusion}

We would like to emphasize the following features of our procedure that distinguishes it from the usual way of constructing the Higgs mechanism:

- Performing the nonlinear stochastic quantization of scalar QED in the approximation of a small coupling of the potential to an internal energy, we arrive at an effective potential equivalent to the symmetry breaking potential usually introduced in the theory of Higgs mechanism. Our starting point was ordinary scalar QED with a potential $V(|\phi|) \sim|\phi|^{2}$ and there was no need to introduce a quartic interaction by hand, as is usually done.

- The parameters $a, b$ of the effective potential have origins in the dynamical structure of the internal energy and its coupling to the potential $V(|\phi|)$. 


\section{References}

[1] C. W. Gardiner: Handbook of Stochastic Methods, Springer, Berlin 2004.

[2] L. Arnold: Stochastische Differentialgleichungen, Oldenbourg, München 1973.

[3] G. Parisi, Y. Wu: Perturbation Theory without Gauge Fixing, Sci. Sin. 24, 483 (1981).

[4] P. Damgaard, H. Hüffel: Stochastic Quantization, Phys. Rep. 152, 227 (1987).

[5] M. Namiki: Stochastic Quantization, Springer, Berlin 1992.

[6] F. Schweitzer, W. Ebeling, and B. Tilch: Complex Motion of Brownian Particles with Energy Depots, Phys. Rev. Lett. 80, 5044 (1998).

[7] U. Erdmann, W. Ebeling, L. Schimansky-Geier, and F. Schweitzer: Brownian Particles far from Equilibrium, Eur. Phys. J. B 15, 105 (2000).

[8] F. Schweitzer: Brownian Agents and Active Particles, Springer, Berlin 2003.

[9] W. Ebeling, Igor M. Sokolov: Statistical Thermodynamics and Stochastic Theory of Nonequilibrium Systems, World Scientific, Singapore 2005.

[10] M. L. Deng, W. Q. Zhu: Stationary Motion of Active Brownian particles, Phys. Rev. E 69, 046105 (2004).

[11] H. Hüffel, G. Kelnhofer: QED Revisited: Proving Equivalence between Path Integral and Stochastic Quantization, Phys. Lett. B 558, 145 (2004). 